\title{
On the height variation of the E-region cowling conductivity - effect of charged dust particles
}

\author{
P. Muralikrishna ${ }^{1}$ and V. H. Kulkarni ${ }^{2}$ \\ ${ }^{1}$ Instituto Nacional de Pesquisas Espaciais - INPE, C.P. 515, 12201-970, S. J. Campos-SP, Brazil \\ ${ }^{2}$ University of Bombay, Mumbai, India
}

Received: 1 September 2005 - Revised: 1 September 2006 - Accepted: 14 September 2006 - Published: 21 November 2006

\begin{abstract}
Height profiles of the Cowling conductivity in the electrojet region, estimated using the atmospheric parameters given by the existing models like CIRA or MSIS and measured electron density profiles, consistently show the heights of the electrojet current intensity peak to be more than $3 \mathrm{~km}$ below those estimated from in-situ measurements using magnetometers on board sounding rockets. Kulkarni and Muralikrishna (2005) attempted to explain this to be due to the effect of neutral dust particles. They reported that neutral dust particles, when they exist in sufficient numbers, can modify the collision parameters, especially in the lower E-region, where dust particles of meteoric origin are known to exist in large numbers, and thereby can modify the Cowling conductivity profile in the electrojet region. This work is extended here to include the effect of charged dust particles. Dust particles can become charged negatively by the attachment of ambient free electrons, and can thus reduce the number density of free electrons especially below the electrojet peak. This can alter the vertical profile of the east-west Hall current driven by the vertical Hall polarization field, thereby causing a net reduction in the electrojet current. Such a decrease in the electrojet current may be observed on the ground magnetograms. This mechanism, as proposed here, can operate only during periods of strong meteor shower activity, when the dust particle density at the assumed deposit height of $103 \mathrm{~km}$ can reach extreme values (for example, $5 \times 10^{4} \mathrm{~cm}^{-3}$ of $1-\mu \mathrm{m}$ diameter dust particles). Such a dense dust layer may even cause a reversal in the normally upward vertical Hall polarization field, within the dust layer, causing a reversal of the electrojet currents below the current peak.
\end{abstract}

Keywords. Ionosphere (Electric fields and currents; Equatorial ionosphere; Particle precipitation)

Correspondence to: $\mathrm{P}$. Muralikrishna

(murali@dae.inpe.br)

\section{Introduction}

Daily variations of the magnetic fields on the ground level in a narrow belt on both sides of the geomagnetic equator are mainly due to the electrojet currents that flow in the Eregion of the Earth's ionosphere. It is now well accepted that the daytime eastward zonal component of the dynamo electric field in the electrojet region drives an eastward Pedersen current and an upward Hall current. Inhibition of this upward Hall current results in the development of a vertical polarization field. This polarization field, in turn, drives an east-west Hall current, thus enhancing the conductivity in the electrojet region to what is known as the Cowling conductivity. This successful theory is thus based on a combination of the Hall $\left(\sigma_{H}\right)$ and the Pederson $\left(\sigma_{P}\right)$ conductivities, which arise in magnetized but collisional plasma in the height region of $90-120 \mathrm{~km}$. The primary electric field is expected to be due to winds and to global dynamo effects in both the ionosphere and the magnetosphere (Forbes, 1981).

Sugiura and Cain (1966) assumed a slab geometry in magneto plasma and studied the current flow pattern. A Cartesian system of coordinates is chosen here, where the $\mathrm{x}$-axis points towards the geomagnetic east, and the y-axis towards the geomagnetic north. Then the z-axis will be in the vertical direction pointing upwards. During daytime, on a quiet day, the primary electric field $E_{0}$ is in the +x-direction and drives the free electrons in the $-\mathrm{x}$-direction and the positive ions in the $+\mathrm{x}$-direction, causing a net Pedersen current of $\sigma_{P} . E_{0}$ in the $+x$-direction. $E_{0}$ also causes an upward Hall drift of both electrons and ions in the $+\mathrm{z}$-direction, producing a net Hall current of $\sigma_{H} \cdot E_{0}$ in the -z-direction, with the positive ions being much heavier than the electrons, the mobility of electrons is much higher than that of the positive ions. Inhibition of this vertical current results in the accumulation of electrons on the top of the slab of finite thickness and positive ions at the bottom, thus generating a polarization field $\mathrm{E}_{p}$ in the $+\mathrm{z}$-direction. A simplified way of looking at this is

Published by Copernicus GmbH on behalf of the European Geosciences Union. 
that the Pedersen current due to the polarization field $\mathrm{E}_{p}$ in the $+\mathrm{z}$-direction is nullified by the Hall current due to $E_{0}$ in the $-\mathrm{z}$-direction, giving the relations:

$\sigma_{P} E_{P}-\sigma_{H} E_{0}=0$

$E_{P}=\frac{\sigma_{H}}{\sigma_{P}} E_{0}$

The net current in the $+\mathrm{x}$-direction is the result of the Pedersen current caused by $E_{0}$ and the Hall current caused by $\mathrm{E}_{P}$. This current can be written as:

$J_{X}=\sigma_{P} E_{0}+\sigma_{H} E_{P}$.

Substituting for $\mathrm{E}_{P}$ from Eq. (2) on can obtain:

$J_{X}=\left[\sigma_{P}+\frac{\sigma_{H}^{2}}{\sigma_{P}}\right] E_{0}$

$J_{X}=\sigma_{C} E_{0}$,

where

$\sigma_{C}=\left[\sigma_{P}+\frac{\sigma_{H}^{2}}{\sigma_{P}}\right]$,

with $\sigma_{C}$ as the Cowling conductivity here. This is a rather simplified picture of what really happens in the electrojet region. It is known that the presence of meridional currents in this region can modify this simple image (Sugiura and Porus, 1969). Several modifications have later been suggested to modify this simple picture (see Richmond, 1991). From Eq. (5), one can see that when the primary zonal electric field $\mathrm{E}_{0}$ does not vary with altitude, the current $\mathrm{J}_{X}$ must have a height variation similar to that of the Cowling conductivity. But in-situ rocket observations of currents from the equatorial stations in India and Peru show that vertical profiles of $J_{X}$ deviate significantly from that of $\sigma_{C}$ (Prakash et al., 1972; Subbraya et al., 1972; Richmond, 1973; Muralikrishna, 1975; Pfaff et al., 1997; Prakash and Subbraya, 1999). Subbaraya et al. (1972) estimated the elctrojet current intensity from rocket measurements of the total magnetic field using proton precession magnetometers, and the electron density by Langmuir probes from Thumba (magnetic dip $0^{\circ} 47^{\prime} \mathrm{S}$ ), India. It is rather well established now that in the electrojet region as height increases the Cowling conductivity as given by Eq. (6) increases with an increase in the electron density to its maximum value at a height which is a few $\mathrm{km}$ above the maximum of the vertical Hall polarization field, and above its maximum it falls with a decrease in the value of the vertical Hall polarization field. The vertical variation of the Hall polarization field can be represented by the $\sigma_{H} / \sigma_{P}$ profile for a primary electric field $E_{0}$ which is constant with altitude, as given by Eq. (2). The electrojet current strength reaches its maximum at a height which is a few $\mathrm{km}$ above the maximum of the east-west Cowling conductivity (Subbaraya et al., 1972; Kulkarni and Muralikrishna, 2005). The current profiles consistently show peaks around the height of $105 \mathrm{~km}$, which differ from the theoretically estimated values (using model atmosphere parameters) by $4-5 \mathrm{~km}$. Subbaraya et al. (1972) attributed this to a probable increase in the primary east-west electric field with height, which we believe is rather unlikely and has no observational support. Kulkarni and Muralikrishna (2005) put forward an alternative explanation based on the effect of neutral meteoric dust on the collision frequencies and thereby on the electrical conductivities, especially in the region below the electrojet peak. The Cowling conductivity profiles, thus modified, seem to be in good agreement with the estimated electrojet current profiles.

\section{Role of charged dust particles}

It is now well known that dust particles play an important role in laboratory and space plasmas (see Merlino and Goree, 2004). As shown by Kulkarni and Muralikrishna (2005), neutral dust particles, when they exist in sufficient numbers, can modify the collision frequencies, and thereby affect the conductivity parameters in the electrojet region. Also, the presence of dust is known to lower the threshold for shear instability onset in gases, when the ratio of dust to air mass density approaches an appreciable fraction of unity (Palaniswamy and Purushotham, 1981). It is logical to assume that dust particles in an ambient plasma can collect electric charges from the plasma. One can estimate the charge on an isolated dust particle in a plasma through different theoretical approaches (Havnes et al., 1990; Merlino and Goree, 2004). One of the methods is to calculate it just as one would calculate the charge on a larger object - for example, an electric probe or a Langmuir probe in the ionospheric plasma. The dust particle attains a negative floating potential such that the net current collected by it from the ambient plasma is maintained at zero level. For a particle with a 1-micrometer radius in a plasma with electron temperature $T_{e}=300^{\circ} \mathrm{K}$, the floating potential of the dust particle, given by the relation $V_{s}=-2.5 k T_{e} / \mathrm{e}$, is about -0.065 Volts, which corresponds to a negative charge of about $7.2 \times 10^{-18}$ Coulombs on the dust. This is equal to about 45 times the charge of an electron.

Ablation of meteors that enter the atmosphere with velocities $\approx 15 \mathrm{~km} \mathrm{~s}^{-1}$ is the most important source of these dust particles of micron sizes (Kornblum, 1969). The concentration of dust particles is greater in the height region $80-90 \mathrm{~km}$. Hunten et al. (1980) show that for a fixed mass input by meteors at altitudes $\geq 90 \mathrm{~km}$ the number of particles of radius $r_{0}$ varies as $r_{0}^{-4}$. Metallic ions, $\mathrm{Fe}^{+}, \mathrm{Mg}^{+}$and $\mathrm{Ca}^{+}$, are known to be integral components in the E-region of the ionosphere.

Observations of persistence of Leonid meteor trails by Drummond et al. (2001) suggest that dust certainly plays a significant role in the ionospheric dynamics. Charged dust 
particles were experimentally detected, for the first time, in the high latitude and were found to be partly responsible for the observed anomalous PMSE (Polar Mesospheric Summer Echoes) events in the backscatter radar, and electron bite outs measured by rockets (Havnes et al., 1996). Large amounts of dust particles with average sizes of about $0.1 \mu$, with number densities of up to several thousands per cubic centimeter and charge densities of the same order were detected. In some parts of the dust layers it was found that the negative charge density on dust was so large that the number of free electrons was significantly reduced there, since the dust acted as a sink for electrons, thus causing electron bite outs. The number of charged dust particles is reported to be 5 to 10 percent of the total dust number density (Gelinas et al., 2005; Lynch et al., 2005). Gelinas et al. (2005) reported that at mid latitude, the dust layer at $90-94 \mathrm{~km}$ and the sodium layer at $94 \mathrm{~km}$ and above were about $10 \mathrm{~km}$ higher in altitude than at high latitudes. Using dust detectors on board sounding rockets Gelinas et al. (1998) measured small (5000-10 $000 \mathrm{amu}$ ) charged dust particles in the tropical mesosphere. They reported the observation of a 5-km thick layer of mostly positively charged dust with negative charges near the bottom of the layer at around $90 \mathrm{~km}$ in the vicinity of an observed sporadic sodium layer and sporadic-E layer. The meteoric dust production model developed by Hunten et al. (1980) predicts an average dust size of $1 \mathrm{~nm}$ at a density of about 1000 per cubic centimeter in the altitude range of $80-90 \mathrm{~km}$. They find that the concentration of dust particles is greater in this height region. It should be noted here that for a dust material density of about $2 \mathrm{gm} \mathrm{cm}^{-3}$, the mass of a $1-\mathrm{nm}$ dust particle is about $5000 \mathrm{amu}$. Mitchell et al. (2001) from rocket experiments conducted from Norway, reported the observation of electron bite outs in the height region of $84-86 \mathrm{~km}$, caused by negatively charged aerosol dust.

One should note here that the size and number density of dust particles in the atmosphere could vary by orders of magnitude from one day to another. Kirkwood and von Zahn (1993) report that the concentration of neutral Fe, assumed to be of meteoric origin, can be as high as $3 \times 10^{4} \mathrm{~cm}^{-3}$ at altitudes as high as 97.5 to $98 \mathrm{~km}$, with a layer thickness as high as $4.2 \mathrm{~km}$. These metallic layers are often associated with the sporadic-E layer. Near the $105-\mathrm{km}$ height the peak densities are of the order of $10^{4} \mathrm{~cm}^{-3}$ (Hunten, 1981; Carter and Forbes, 1999). Hunten et al. (1981) suggest that a maximum deposition of dust and smoke particles due to meteors occurs in the height region of 70-90 km. McNeil et al. (1996) model a distribution of magnesium ions in the ionosphere, assuming that the main source of these particles is the meteor ablation. A quantitative analysis of the effect of charged dust particles on the electrojet currents needs precise information on the height distribution, as well as the size distribution of the dust particles. This information is still lacking.

\section{Theoretical formulation and discussion}

The effect of negatively charged dust particles, when they exist in sufficient numbers, on the electrojet intensity, is discussed here. The role of these dust particles is to reduce the electron number density. The Pedersen and Hall conductivities in the electrojet region are mainly due to electrons, since they have much larger mobilities compared to positive ions that are much heavier. Any variation in electron density due to charged dust particles would, therefore, affect the conductivities. The presence of the $10-30-\mathrm{nm}$ size dust particles in the upper and lower mesosphere is a regular phenomenon (Berger and von Zahn, 2002). At normal electron temperatures one can see that these dust particles can attract at most one electron. It should be noted here that these dust particles have masses in the range of 50000 to $150000 \mathrm{amu}$ and thereby are still much heavier than the positive ions. Their mobility in the electrojet region can be assumed to be practically zero. If they exist in large numbers of the order of $10^{4}$ per cubic centimeter, they can still affect the Hall current, especially in the height region below the electrojet peak, where the normal electron densities are of the order of $10^{4} \mathrm{~cm}^{-3}$. During days of meteor showers, one can expect dust particles of a wide spectrum of sizes, ranging from a few nanometers to a few microns or even bigger, in sufficient numbers as to attract all the electrons in the height region below the electrojet peak. This can cause a reduction in or even a reversal of the normal electrojet current in this height region, through the suppression of the Hall conductivity of electrons. Such a reduction in the electrojet currents may be observed as a decrease in the $\mathrm{H}$ component of the geomagnetic field. If the charged dust layer exhibits a double polarity, positive on top and negative at bottom (Gelinas et al., 1998), the nature of the Hall polarization field and the vertical structure of the electrojet current can become complex. If this results in the reversal of the vertical Hall polarization field in the region of the dust layer, one can even expect the reversed currents to be sufficiently large as to cause the phenomenon of counter electrojet, as identified in the ground magnetograms. The present attempt is to have a qualitative and not quantitative picture of the effect of charged dust particles on the normal electrojet current, using some simplifying assumptions. In the theoretical formulation presented here the following assumptions are made:

- The number density of dust particles of mean diameter around $1 \mu$ can be as high as $10^{4}$ to $10^{5} \mathrm{~cm}^{-3}$, occasionally when there are meteor shower events and about $10 \%$ of these particles may become negatively charged by capturing more than 6 electrons each from the ambient plasma.

- In the presence of charged dust particles the Eregion plasma continues to maintain its state of 


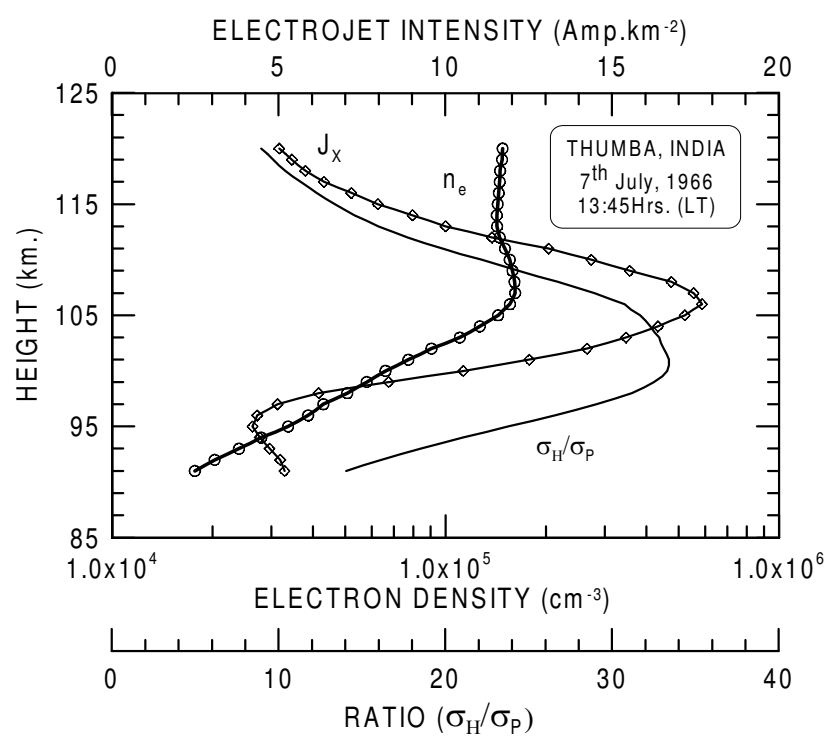

Fig. 1. Height profiles of the electron density and electrojet current intensity estimated from in-situ measurements made from Thumba, India at 13:45 LT on 7 July 1966 (Flight 20.05), compared with the model estimate of the ratio of Hall to Pedersen conductivity profile.

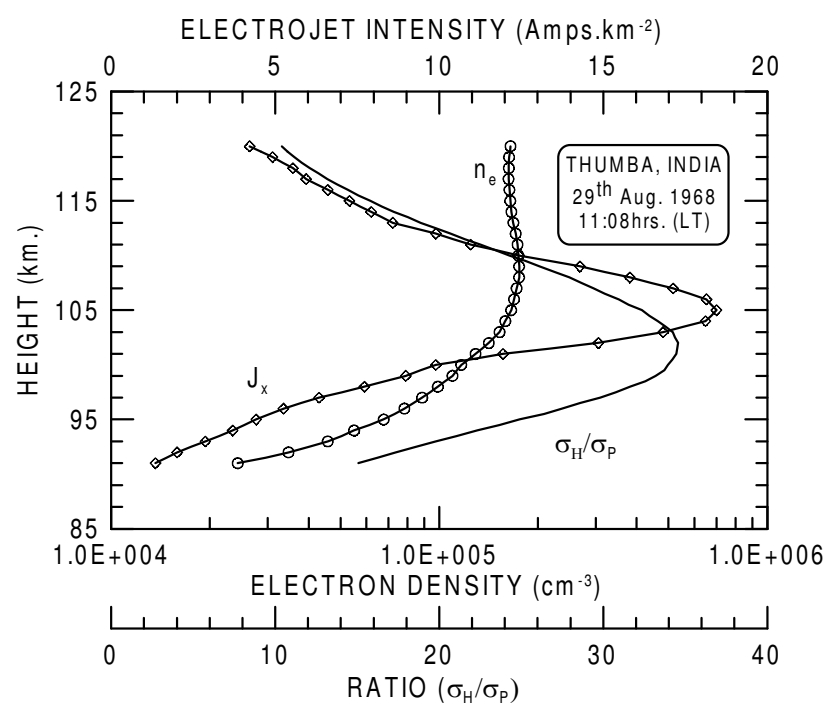

Fig. 2. Height profiles of the electron density and electrojet current intensity estimated from in-situ measurements made from Thumba, India at 11:08 LT on 29 August 1968 (Flight 20.06), compared with the model estimate of the ratio of Hall to Pedersen conductivity profile.

quasi-neutrality, satisfying the relation:

$n_{i}=n_{e}^{\prime}+n_{d e}$,

where $n_{e}^{\prime}$ is the number density of free electrons, $n_{i}$ is the number density of positive ions and $n_{d e}$ is the num-

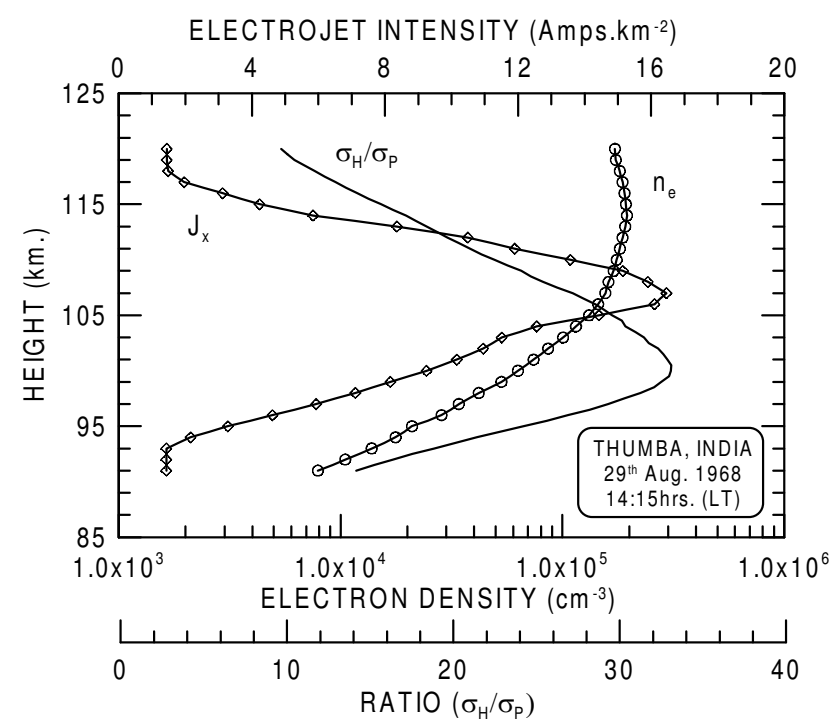

Fig. 3. Height profiles of the electron density and electrojet current intensity estimated from in-situ measurements made from Thumba, India at 14:15 LT on 29 August 1968 (Flight 20.07), compared with the model estimate of the ratio of Hall to Pedersen conductivity profile.

ber of electrons attached to dust particles per unit volume. In the absence of charged dust particles, it follows that $n_{e}^{\prime}=n_{e}=n_{i}$.

- In the height region below the electrojet peak, where dust particles are dominantly observed, the mobilities of the free electrons, positive ions and negatively charged dust particles roughly satisfy the inequality condition:

$\mu_{e}>\mu_{i} \gg \mu_{d}$.

- The mobility of charged dust particles that are much heavier than the positive ions is assumed to be negligible $\left(\mu_{d}=0\right)$ when compared with those of electrons and positive ions.

- The number of negative ions is negligible.

Figures 1 to 3 show the height profiles of the ratio $\sigma_{H} / \sigma_{P}$, which is a measure of the vertical Hall polarization field, as seen from Eq. (2), estimated from the MSISE-90 atmospheric model, and the ambient electron density measured using rocket-borne experiments (also given in the figures) conducted from the equatorial station Thumba, India (Subbaraya et al., 1972; Muralikrishna, 1975). Also given in the figures are the electrojet current intensities estimated from insitu observations of the electrojet current. Although Figs. 1 to 3 represent measurements made on different days or times, Fig. 1 represents the electrojet currents at 13:45 LT, Fig. 2 those at 11:08 and Fig. 3 those at 14:15, thus representing the daytime electrojet at different intensities compared with its daytime maximum intensity. 
In the height region above the electrojet peak, where the dust particles are practically absent, the polarization field $\boldsymbol{E}_{P}$ enhances the east-west electrojet current, as explained through Eqs. (4) to (6). In this region the Hall drifts of both free electrons and positive ions are in the $\boldsymbol{E}_{P} \times \boldsymbol{B}$ direction ( - x-direction), with that of electrons being much larger than that of the ions, thereby causing a net Hall current in the $-\boldsymbol{E}_{P} \times \boldsymbol{B}$ direction (+x-direction). But in the region below the electrojet peak, where the charged dust particles remove a large number of free electrons from the ambient plasma, the picture is different. Though the net current density is due to the Pedersen and Hall drifts of electrons and positive ions, the number densities of electrons and positive ions are not equal in this region. The relative contributions of electrons and positive ions to this net current depend very much on their relative number densities. The acquisition of electrons by the dust particles depends on their number density and size distribution. In the extreme case when all the free electrons are attached to the dust particles, the net Hall current is determined by the Hall drift of positive ions only and will be in the $-\mathrm{x}$-direction, opposite to that of the normal electrojet currents. Though this represents a small fraction of the total Hall current, its effect is to reduce the normal current in the lower electrojet region or even to cause a reversal in it. This situation can be mathematically represented as shown below.

The contribution to the Hall conductivity by the electrons and positive ions is represented by $\sigma_{H e}$ and $\sigma_{H i}$, respectively, and is given by the relations (see Kulkarni and Muralikrishna, 2005):

$$
\begin{aligned}
\sigma_{H e} & =+\frac{n_{e}^{\prime} e^{2} \omega_{e}}{m_{e}\left(\omega_{e}^{2}+v_{e}^{2}\right)} \\
\sigma_{H i} & =-\frac{n_{i} e^{2} \omega_{i}}{m_{i}\left(\omega_{i}^{2}+v_{i}^{2}\right)},
\end{aligned}
$$

where $e$ is the charge of an electron, $m_{e}$ and $m_{i}$ are the masses, $\omega_{e}$ and $\omega_{i}$ are the gyrofrequencies and $v_{e}$ and $v_{i}$ are the collision frequencies of electrons and positive ions, respectively, $n_{e}^{\prime}$ is the number density of free electrons in the ambient plasma and $n_{i}$ is the number density of positive ions. The positive sign in Eq. (7) shows that the Hall drift of electrons is in the $-\mathrm{X}$-direction, causing a positive current in the $+\mathrm{x}$-direction. The negative sign in Eq. (8) shows that the Hall drift of positive ions, also in the $-\mathrm{x}$-direction, causes a negative current in the $+\mathrm{x}$-direction. It should be noted here that the ion Hall current is orders of magnitude less than the electron Hall current. One must also remember here that $n_{e}^{\prime}<n_{i}$, since a large number of free electrons are attached to the dust particles and the plasma still remains quasi-neutral. Under these conditions one can write the relation:

$n_{i}=n_{e}^{\prime}+N . n_{d}$,

where $N$ is the average number of electrons attached to a dust particle and $n_{d}$ is the number density of charged dust particles.

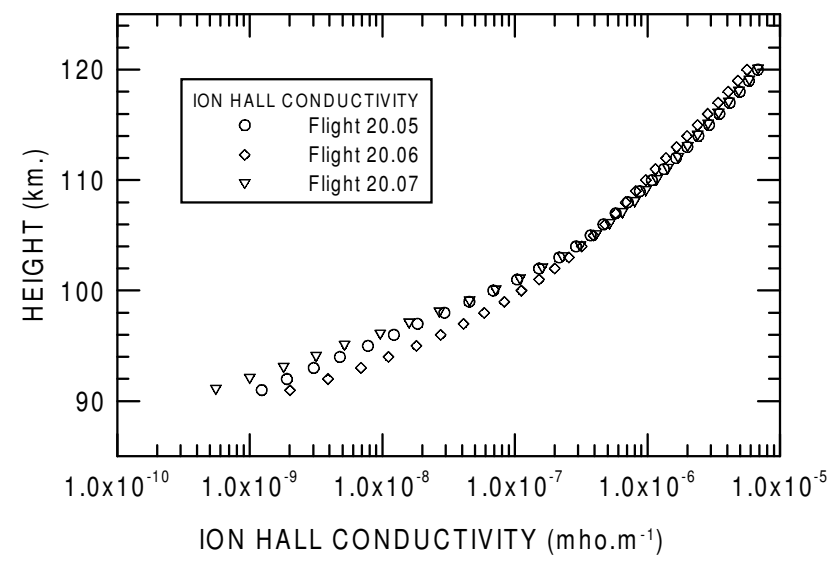

Fig. 4. Height profile of the magnitude of the ion Hall conductivity $\sigma_{\mathrm{Hi}}$ estimated for the three cases represented in Figs. 1 to 3 for the rocket flights 20.05, 20.06 and 20.07, respectively.

In the height region where there are no charged dust particles, $n_{e}^{\prime}=n_{e}$ and the contribution of electrons to Hall conductivity is much larger than that of positive ions, thus causing a net Hall current in the $+x$-direction. In the height region where the number density of charged dust particles is high, the contribution of electrons to the Hall conductivity can be much smaller, of the order of, or larger than that of, positive ions, depending on whether $\sigma_{H e} \ll \sigma_{H i}, \sigma_{H e}<\sigma_{H i}$ or $\sigma_{H e}=\sigma_{H i}$. This, in turn, depends on whether $n_{e}^{\prime} \ll n_{i} ; n_{e}^{\prime}<n_{i}$; or $n_{e}^{\prime}=n_{i}$.

The height variation of the Hall conductivity of ions estimated through Eq. (7) is compared in Fig. 4 for the three electron density profiles presented in Figs. 1 to 3 . Under the conditions governed by the simplifying assumptions made here, the Hall conductivity of positive ions is practically zero below $90 \mathrm{~km}$, and is significant and comparable with the Hall conductivity of the electrons only in the height region above $95 \mathrm{~km}$. This situation is the same for all three cases shown in Figs. 1 to 3 . If a charged dust layer is present in the height region between $95 \mathrm{~km}$ and the peak of the electrojet, it can definitely cause a significant reduction in or even a reversal of the net Hall current in this height region. With the Hall conductivity of ions being orders of magnitude less than that of electrons, the reversed currents caused by ions through the process explained above may be insignificant compared with the Hall current caused by the electrons.

\subsection{Dust layer approximation}

To make a quantitative estimate of the effect of a charged dust layer on the electrojet currents, a dust layer with a uniform dust size of 1 micrometer in diameter is assumed here. The maximum number density of the dust particles is assumed to be $5 \times 10^{4} \mathrm{~cm}^{-3}$ at the assumed dust deposit height of $103 \mathrm{~km}$ (below the electrojet peak), decreasing exponentially to zero at $93 \mathrm{~km}$. The floating potential of a dust particle 


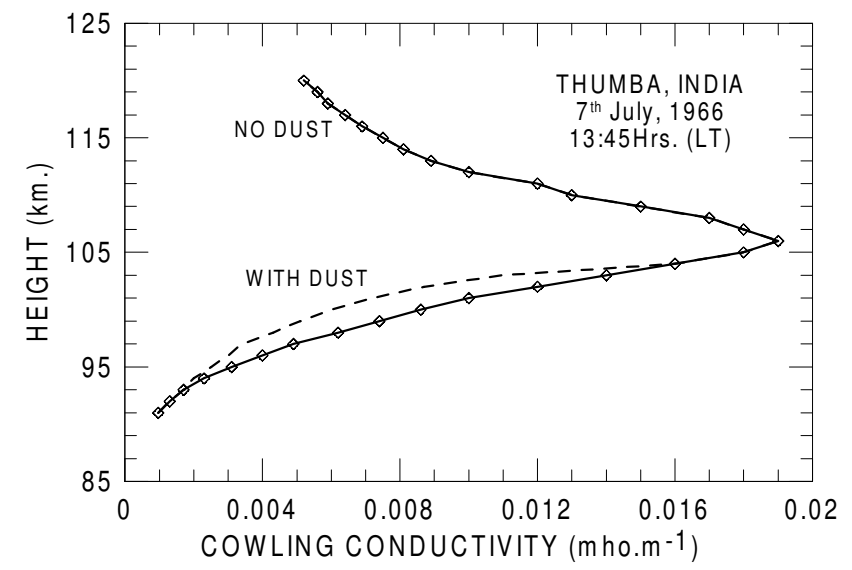

Fig. 5. Height profile of the Cowling conductivity under conditions of maximum electrojet currents on 7 July 1966 at 13:45 LT with and without an assumed charged dust layer.

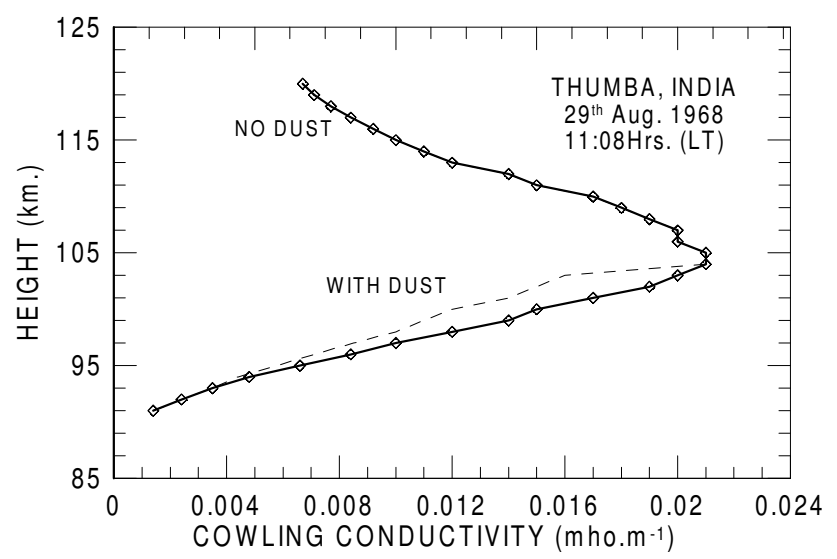

Fig. 6. Height profile of the Cowling conductivity on 29 August 1968 at 11:08 LT with and without an assumed charged dust layer.

of 1 micrometer in diameter, as estimated from the relation $V_{s}=-2.5 k T_{e} / \mathrm{e}$ (Sect. 2), is about -0.016 Volts, which is more than 6 times the electronic charge. Assuming that about $10 \%$ of these dust particles become negatively charged (Gelinas et al., 2005), one can see that the number of electrons captured by the dust particles is more than $3 \times 10^{4}$ per cubic centimeter at the dust deposit height of $103 \mathrm{~km}$. This, in fact, is close to the number density of free electrons in the bottom side of the E-region, under normal conditions. Since the surface area of the dust particle varies as the square of its radius, dust particles with a diameter of $2 \mu \mathrm{m}$ can capture four times more the number of electrons.

Height profiles of the Cowling conductivity with and without the presence of a dust layer, estimated for the three different electrojet conditions shown in Figs. 1 to 3, are estimated using the above dust model and presented in Figs. 5 to 7. The decrease in the Cowling conductivity that is a measure of the electrojet current intensity, in the region of the dust layer

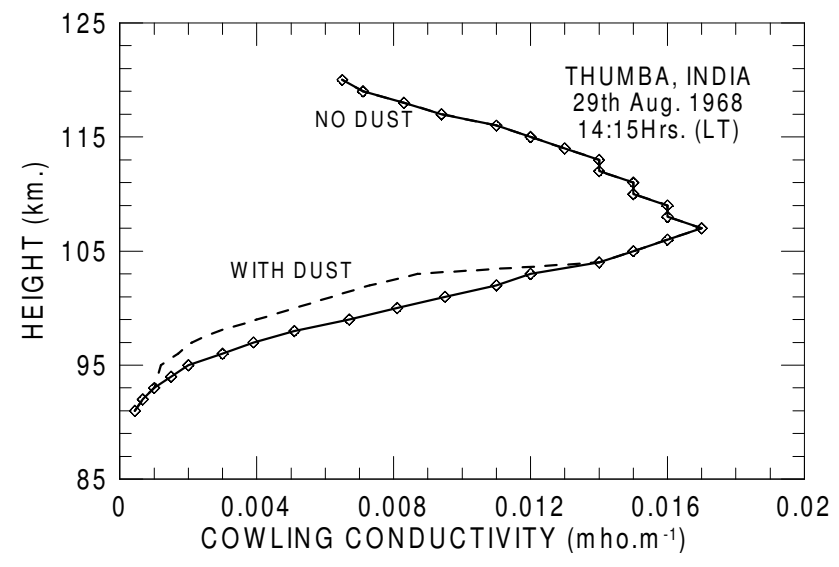

Fig. 7. Height profile of the Cowling conductivity on 29 August 1968 at 14:15 LT with and without an assumed charged dust layer.

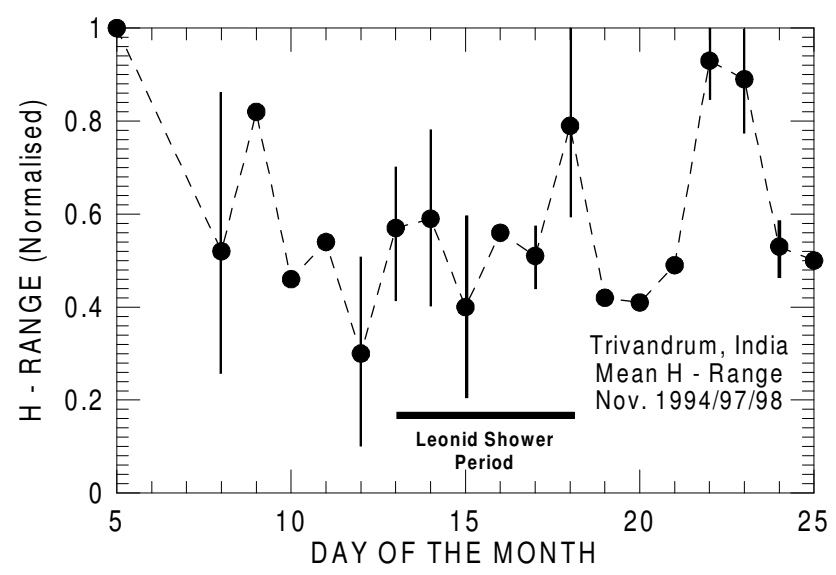

Fig. 8. Variation in the daily range of the horizontal component of the geomagnetic field at Trivandrum, India on quiet days during the month of November, averaged for the years 1994, 1997 and 1998. Vertical lines in the figure show the data spread. Also indicated in the figure is the Leonid meteor shower period.

(93-103 km), can be clearly seen from these figures. Under conditions of electrojet peak, as shown in Fig. 7, the decrease in the Cowling conductivity is less pronounced than under conditions of a weaker electrojet, shown in Fig. 6 (before noon) and Fig. 7 (after noon). Depending on the actual dust density, the diameter and distribution of this decrease in the Cowling conductivity can be more or less than that shown in Figs. 5 to 7. Such a decrease in the electrojet currents, if it exists, must leave its signature in the ground level geomagnetic variations. The mean daily range of the horizontal component of the geomagnetic field at the equatorial station Trivandrum, India, close to Thumba, India, is shown in Fig. 8, from where the rocket measurements presented in Figs. 1 to 3 were made. Estimated values of the mean normalized daily range (difference between the daytime maximum and the nighttime base level) of the horizontal component of the geomagnetic 
field for quiet days between 5 and 25 November for the years 1994, 1997 and 1998 are presented in the figure. The vertical line associated with the points indicates the spread of the normalized range observed for the three years. The absence of the vertical line with some points indicates that the point corresponds only to a single observation. The Leonid shower periods during the three years considered here extended from around 13 to 18 November and is also shown in the figure. Associated with the intense Leonid shower period, one can see a general decrease in the mean H-range, indicating a corresponding decrease in the integrated electrojet current intensity. One should remember here that a decrease in the electrojet currents can be attributed to other control parameters, like the intensity of the driving dynamo electric field that can vary from day to day. However, such a decrease in the electrojet currents can also be attributed to the mechanism proposed here through the effects of charged dust particles, and further studies are needed to conclusively establish this hypothesis.

\subsection{Counter electrojet}

It should be noted here that in the model approach discussed above the only physical effect considered is that of the capture of free electrons by the dust particles. The vertical polarization electric field is assumed to be given by the simple relation represented by Eq. (2). The effect of the charged dust layer on the height profile of the vertical polarization field is therefore neglected. Under these assumptions the intensity of the reversed currents is limited to that caused by the ion Hall current, which is rather insignificant. The presence of a charged dust layer with significant thickness can distort the vertical polarization field and can even create height regions where the vertical polarization field is reversed from its normally upward direction. This, in turn, can cause a reversal in the electrojet currents in those height regions. In addition to an improved knowledge on the size and number density distribution of the meteoric dust, a detailed theoretical approach is needed to make any quantitative estimate of the effect of charged dust particles on producing a counter electrojet.

Thus, considering the effect of charged dust particles on the electrojet currents, one can have the following different situations:

1. The number of charged dust particles is negligibly small. This leaves the vertical profile of the Cowling conductivity unaltered and one has the situation of normal electrojet currents flowing in the $+x$-direction throughout the vertical extent of the electrojet region.

2. The number of charged dust particles is considerable, especially in the height region below the electrojet peak. Even if one assumes that this charged dust layer does not affect the vertical Hall polarization field, it can reduce the Hall currents due to electrons in this height region, thereby reducing the electrojet current in the $+\mathrm{x}-$ direction.

3. The number of charged dust particles is very high in the height region below the electrojet peak. This practically reduces the Hall currents due to electrons to zero in this height region. Such a large decrease in the electrojet currents may cause a decrease in the normal daily range of the horizontal component of the geomagnetic field.

4. The formation of a charged dust layer can alter the height profile of the vertical polarization field, producing height regions of a reversed vertical polarization field, thereby causing a reversal of the normal electrojet current in these height regions. This may result in the flow of a partial or full counter electrojet.

What is given here is a very simplified picture of the effect of charged dust particles. The real image can be much more complex. The charged dust layer can even modify the vertical structure of the Hall polarization field. One can complicate the situation further by taking into account the effect of positively charged dust particles (attributed to photoemission), as well. Under these conditions it is possible that some of the observed counter electrojet events are associated with reversals in the electron drift.

Apart from all these considerations we would like to present below some of the established facts about the phenomenon of counter electrojet, most of which are in support of our present hypothesis that negatively charged dust particles can play a very significant role in distorting the electrojet currents especially below its maximum.

- Counter electrojets do not occur very often, but when they do, they normally continue for a few days, probably due to the continuous effect of the meteoric dust particles which may take several days to precipitate to lower altitudes.

- The reversed currents are never observed in the upper E-region, but always in the lower E-region.

- Strong counter electrojets are normally associated with a blanketing type of sporadic-E layer, also always confined to the lower E-region.

- Nanometer size particles with single electrons may still be present in large numbers in the lower E-region. Even if the number of electrons in these dust particles is small, their mass is much higher than that of positive ions, and thereby their Hall mobility is practically negligible compared to that of electrons or ions.

- A counter electrojet can show a Lunar tide dependence if it has a strong association with the heavier dust particles, which may take part in tidal oscillations. 
- There is no problem of finding a return path for the reversed currents, since these may flow as large eddy currents, depending on the latitudinal and longitudinal distribution of charged dust particles.

- A counter electrojet occurs more frequently in the morning and afternoon hours (see Rastogi, 1974; Mayaud, 1977), when the normal electrojet currents are rather weak. When the normal electrojet currents are weak, the reversed currents can dominate.

A coordinated measurement with multiple experiment payloads alone can resolve this complicated problem.

\section{Conclusions}

- Negative charging of dust particles can reduce the number density of ambient free electrons in the electrojet region, especially below the electrojet peak, where the number density of dust particles of meteoric origin is known to be high.

- The reduction in the number density of free electrons in the height region dominated by negatively charged dust particles could cause a reduction in or even a reversal of the normal electrojet currents.

- Observation of a counter electrojet in the morning or afternoon hours when the normal electrojet currents are rather weak supports the present thesis.

- Observation of normal and/or blanketing types of sporadic-E layers in association with strong counter electrojet events, is also in agreement with the present hypothesis that negatively charged dust particles may reverse the vertical polarization field and can cause current reversals in the height region below the electrojet peak. These charged dust particles when in abundance can cause a sporadic-E layer in the ground ionograms.

Acknowledgements. The authors are thankful to the director of INPE for his encouragement. The work reported here was partially supported by the FNDCT under contract FINEP 537/CT and by the Conselho Nacional de Desenvolvimento Científico e Tecnológico CNPq under process 301805/2004-0. V. H. Kulkarni gratefully acknowledges the support of CNPq, Brazil through the PCI fellowship and the support of FAPESP through process no. 1999/00437-0.

Topical Editor M. Pinnock thanks R. Rajaram and another referee for their help in evaluating this paper.

\section{References}

Berger, U. and von Zahn, U.: Icy particles in the summer mesopause region: 3-D modeling of their environment and 2-D modeling of their transport, J. Geophys. Res., 107(A11), 1366, doi:10.1029/2001JA000316, 2002.
Carter, L. N. and Forbes, J. M.: Global transport and localized layering of metallic ions in the upper atmosphere, Ann. Geophys., 17, 190-209, 1999, http://www.ann-geophys.net/17/190/1999/.

Drummond, J. D., Grime, B. W., Gardner, C. S., Liu, A. Z., Chug, X., and Kane, T. J.: Observations of persistent Leonid meteor trails, 1. Advection of the "Diamond Ring", J. Geophys. Res., 106, 21 517-21 524, 2001.

Forbes, J. M.: The equatorial electrojet, J. Geophys. Res., 19, 469504, 1981.

Gelinas, L. J., Lynch, K. A., Kelley, M. C., Collins, S., Baker, S., Zhou, Q., and Friedman, J. S.: First observation of meteoric charged dust in the tropical mesosphere, Geophys. Res. Lett., 25(21), 4047-4050, 1998.

Gelinas, L. J., Lynch, K. A., Kelley, M. C., Collins, R. L., Widholm, M., Rau, D., Mac Donald, E., Liu, Y., Ulwick, J., and Mace, P.: Mesospheric charged dust layer: implication for neutral chemistry, J. Geophys. Res., 110, A01310, doi:10.1029/2004JA010503, 2005.

Havnes, O., Aanesen, T. K., and Melands $\varnothing$, F.: On dust charges and plasma potentials in a dusty plasma with dust size distribution, J. Geophys. Res., 95(A5), 6581-6585, 1990.

Havnes, O., Trøim, J., Blix, T., Mortensen, W., Naesheim, L. I., Thrane, E., and Tønnesen, T.: First detection of charged dust particles in the earth's mesosphere, J. Geophys. Res., 101(A5), 10 839-10 847, 1996.

Hunten, D. M.: A meteor ablation model of the sodium and potassium layers, Geophys. Res. Lett., 8, 369-372, 1981.

Hunten, D. M., Turco, R. P., and Toon, O. B.: Smoke and dust particles of meteoric origin in the mesosphere and stratosphere, J. Atmos. Sci., 37, 1342-1357, 1980.

Kirkwood, S. and von Zahn, U.: Formation mechanism of lowaltitude Es and their relationship with neutral Fe layers - Results from METAL campaign, J. Geophys. Res., 98(A12), 21 549$21561,1993$.

Kornblum, J. J.: Micrometeoroid interaction with the atmosphere, J. Geophys. Res., 74, 1893-1919, 1969.

Kulkarni, V. H. and Muralikrishna, P.: The role of dusts on the equatorial electrojet currents, J. Atmos. Solar Terr. Phys., 68/2, 228235, doi:10.1016/j.jastp.2005.10.007, 2005.

Lynch, K. A., Gelinas, L. J., Kelley, M. C., Collins, R. L., Widholm, M., Rau, D., Mac Donald, E., Liu, Y., Ulwick, J., and Mace, P.: Multiple sounding rocket observations of charged dust in the polar winter mesosphere, J. Geophys. Res., 110, A03302, doi:10.1029/2004JA010502, 2005.

Mayaud, P. N.: The equatorial counter electrojet, A review of its geomagnetic aspects, J. Atmos. Terr. Phys., 39, 1055-1070, 1977.

Merlino, L. and Goree, J. A.: Dusty plasmas in the laboratory, industry and space, Phys. Today, 57(7), 32-38, 2004.

McNeil, W. J., Lai, S. T., and Murad, E.: A model for meteoric magnesium in the ionosphere, J. Geophys. Lett., 101, 5251-5260, 1996.

Mitchell, J. D., Croskey, C. L., and Goldberg, R. A.: Evidence of charged aerosols and associated meter-scale structure in identified PMSE/NLC regions, Geophys. Res. Lett., 28(8), 14231426, 2001.

Muralikrishna, P.: Studies in Equatorial Aeronomy - Morphology of the Equatorial Electrojet, Ph. D. Thesis, Gujarat University, 1975 . 
Palaniswamy, V. I. and Purushotham, C. M.: Stability of shear flow of stratified fluids with fine dust, Phys. Fluids, 24, 1224, 1981.

Pfaff Jr. R. F., Acuña, M. H., Marionni, P. A., and Tivedi, N. B.: DC polarization electric field, current density, and plasma density measurements in the daytime equatorial electrojet, Geophys. Res. Lett., 24, 1667-1670, 1997.

Prakash, S. and Subbaraya, B. H.: Upper atmospheric studies in India with rocket borne techniques, Published in Space Research in India: accomplishments and prospects, PRL alumni association, Ahmedabad, August, 137-178, 1999.

Prakash, S., Subbaraya, B. H., and Gupta, S. P.: Rocket measurement of ionization irregularities in the equatorial ionosphere at Thumba and identification of plasma instabilities, Indian J. Radio Space Phys., 1, 72-80, 1972.
Rastogi, R. G.: Westward equatorial electrojet during daytime hours, J. Geophys. Res., 79, 1503-1512, 1974.

Richmond, A. D.: Equatorial electrojet II. Use of the model to study the equatorial ionosphere, J. Atmos. Terr. Phys., 35, 1105, 1973.

Richmond, A. D.: Modeling of equatorial ionospheric electric fields, J. Atmos. Terr. Phys., 57, 1103-1114, 1991.

Subbaraya, B. H., Muralikrishna, P., Sastry, T. S. G., and Prakash, S.: A study of the structure of electrical conductivities and the electrostatic field within the equatorial electrojet, Planet. Space Sci., 20, 47-52, 1972.

Sugiura, M. and Cain, J. C.: A model equatorial electrojet, J. Geophys. Res., 71, 1869-1877, 1966.

Sugiura, M. and Poros, D. J.: An improved model equatorial electrojet with a meridional current system, J. Geophys. Res., 74, 4025-4034, 1969. 